\title{
Medicina Interna e organizzazione ospedaliera per intensità di cure: la posizione di FADOI Emilia Romagna
}

\author{
Internal Medicine and hospital organization based on treatment intensity: \\ the position of FADOI Emilia Romagna
}

\section{Messaggi chiave}

FADOI Emilia Romagna, associazione degli internisti ospedalieri, si esprime a favore di una rimodulazione dell'organizzazione ospedaliera secondo il principio della "intensità di cure".

- Un assetto organizzativo strutturato "per intensità di cure" deve essere applicato in maniera flessibile in considerazione delle diverse caratteristiche della struttura nella quale viene costruito.

- L'organizzazione per intensità di cure non abolisce l'articolazione in diverse Unità Operative, ma ne struttura in maniera diversa gli assetti organizzativi.

- I livelli sui quali può essere articolata questa organizzazione sono preferibilmente tre: un'area ad alta intensità, un'area a intensità intermedia, un'area per pazienti post-acuti.

- La diversificazione per aree deve prevedere una differente presenza di personale medico e infermieristico e una differente dotazione tecnologica.

- È necessario definire criteri e percorsi per l'allocazione dei pazienti nelle diverse aree.

- È necessaria una precisa definizione delle responsabilità, nell'ambito delle quali un ruolo particolare, specie di coordinamento delle varie figure professionali afferenti, deve essere svolto dal medico internista, per le sue competenze specifiche.

- Le responsabilità di tipo clinico e gestionale per ogni singolo paziente sono in a carico all'Unità Operativa alla quale il paziente è assegnato.

\section{Premessa}

Negli ultimi vent'anni è profondamente cambiata la tipologia di pazienti afferenti ai nostri ospedali. Oggi si tratta prevalentemente di pazienti anziani, complessi, affetti da polipatologie e destinatari di trattamenti altrettanto complessi. Parallelamente, lo sviluppo tecnologico ha cambiato in maniera significativa le modalità di assistenza di numerose patologie acute, talora con livelli di evidenza molto significativi. Inoltre, l'opinione pubblica è decisamente più attenta alle modalità e ai livelli di assistenza e di cura erogati dalle diverse strutture.

Tutto questo rende necessario ripensare e rendere più attuali i modelli organizzativi presenti all'interno dei nostri ospedali e della nostra rete ospedaliera. Per quanto riguarda la riorganizzazione degli ospedali, il modello organizzativo cosiddetto "per intensità di cure", coniugato con il concetto di ospedali "in rete" secondo il principio "hub and spoke" sembrerebbe più aderente a tali nuove esigenze.

Il modello assistenziale per intensità di cure coinvolge in maniera significativa le Unità Operative di Medicina Interna, alle quali afferisce la maggior parte dei pazienti corrispondenti alle tipologie sopra elencate.

Riteniamo pertanto opportuno, come medici internisti ospedalieri coinvolti in questi processi, esprimere la nostra posizione su tale modello organizzativo assistenziale e su come esso possa essere costruito e strutturato all'interno dei nostri ospedali.

Riteniamo il modello per intensità di cure più rispondente alle esigenze della medicina moderna, in quanto:

- consente di distribuire i pazienti in relazione alla loro severità e criticità, e non semplicemente per singola patologia; 
- considera il paziente nella sua interezza e nella sua complessità e polipatologicità e prevede approcci integrati e multidisciplinari;

- prevede l'utilizzo di supporti tecnologici appropriati alla situazione clinica di ogni singolo paziente.

\section{Dove può essere applicato il modello per intensità di cure?}

Non vi è un ambito specifico per la collocazione e la realizzazione di questo modello. Riteniamo che il modello possa e debba essere applicato con la massima flessibilità, adattandosi pienamente alle caratteristiche della struttura e/o dell'area nel cui contesto viene attuato. Potranno pertanto esservi modelli strutturati:

- su un intero ospedale;

- in ambito dipartimentale;

- all'interno di un'unica Unità Operativa di adeguate dimensioni;

- in macroaggregazioni di aree e/o discipline anche non omogenee.

\section{Quali sono le modalità per una sua efficace applicazione?}

Quali punti qualificanti, determinanti e necessari per la realizzazione di questo modello assistenziale abbiamo identificato:

- la corretta individuazione di

- livelli assistenziali sui quali strutturare il modello,

- tipologie di pazienti ammissibili presso le diverse aree;

- l'allocazione delle risorse (in termini di personale, di tipologia strutturale dell'area e di tecnologie) ben definita e differenziata a seconda dei diversi livelli assistenziali;

- la condivisione delle modalità di assistenza tra medici accettanti e medici di Pronto Soccorso già a partire dall'arrivo in Pronto Soccorso;

- la definizione di precise responsabilità che, a cascata, regolino la gestione delle diverse aree, le modalità operative e di collaborazione tra i diversi professionisti e le diverse équipe che vi afferiscono.

\section{Quali sono i livelli assistenziali ottimali e qual è la tipologia dei pazienti ammissibili?}

Riteniamo che $\mathrm{i}$ livelli assistenziali ottimali di degenza sui quali costruire questo modello possono essere i seguenti:

- area di degenza a più alta intensità di cure: fase di malattia severa in condizione di non stabilità clinica che non necessiti di un accesso a un'Unità di Terapia Intensiva rianimatoria;

- area di degenza per pazienti acuti: fase di malattia ancora in acuzie, ma con minor grado di criticità/instabilità;

- area di degenza a più bassa intensità di cure: paziente sufficientemente stabilizzato, ma ancora a potenziale elevato rischio di ricadute o complicanze e/o necessitante di terapia ospedaliera "complessa". Tale paziente potrebbe anche essere definito come "post-acuto".
Per queste aree deve essere prevista un'adeguata e ben definita collocazione geografica. Le diverse aree devono essere collocate preferibilmente in spazi adiacenti e opportunamente collegati.

Riteniamo che le Unità di Terapia Intensiva non debbano essere comprese all'interno di questo modello, ma debbano rimanere aree autonome e separate, anche se, ovviamente, collegate in maniera adeguata alle altre aree dell'ospedale nel quale sono situate.

\section{Quali risorse devono essere previste (in termini di personale, di tipologia strutturale dell'area e di tecnologie) a seconda dei diversi livelli assistenziali?}

Devono essere previste una dotazione tecnologica e una presenza di personale, sia medico sia infermieristico, diversificate a seconda delle aree.

Per ciascuna area e in particolare per l'area cosiddetta "ad alta intensità" deve essere definita una dotazione tecnologica adeguata alle necessità della tipologie di pazienti ammessi.

Nell'area ad alta intensità la presenza medica deve differenziarsi e caratterizzarsi per un maggior numero di accessi programmati, opportunamente documentati; deve essere prevista anche una presenza incrementata di infermieri, opportunamente addestrati e preparati all'utilizzo delle tecnologie presenti, in misura intermedia tra le aree di tipo intensivistico e le aree di degenza ordinaria. Per le aree a minore intensità, cosiddette "per post-acuti", la presenza di professionalità mediche e di infermieri specializzati può essere lievemente inferiore rispetto alle altre aree del modello, mentre la presenza di personale infermieristico e ausiliario deve essere opportunamente proporzionata alle peculiari necessità assistenziali di base (mobilizzazione, cura della persona, igiene alimentare, ecc.).

\section{Quali rapporti tra reparti, Pronto Soccorso e accettazione?}

Riteniamo che un modello assistenziale strutturato per intensità di cure necessiti di una stratificazione della severità e delle caratteristiche cliniche dei pazienti già all'ammissione in ospedale.

Deve quindi essere rafforzata e consolidata l'interfaccia tra Pronto Soccorso, accettazione medica e reparti di degenza.

Devono essere strutturati percorsi clinico-assistenziali per le principali patologie trattate che prevedano già a partire dalle fasi di accettazione snodi decisionali non solo di tipo diagnostico e/o terapeutico, ma anche in merito alla stratificazione di severità, con conseguente definizione di criteri condivisi per l'allocazione dei pazienti all'interno di una delle diverse aree di intensità.

Auspichiamo che le aree di Osservazione Breve Intensiva, attualmente presenti presso il Pronto Soccorso di vari ospedali, diventino aree potenzialmente condivise tra il Pronto Soccorso e i medici dei reparti di accettazione, specialmente di area internistica, al fine di migliorare l'appropriatezza dei 
ricoveri e la corretta allocazione di ciascun paziente a uno dei diversi livelli di intensità.

\section{Come attribuire responsabilità e ruoli?}

Riteniamo che la definizione delle responsabilità sia prerequisito determinante ai fini di un corretto ed efficace funzionamento di questo sistema organizzativo.

L'assetto organizzativo deve prevedere una responsabilità "ultima" di tipo apicale (direttore di struttura complessa o struttura semplice dipartimentale) alla quale, a cascata, conseguano altre e ben definite sottoresponsabilità (di un'area, di un'aggregazione di letti all'interno di un'area ecc.).

La responsabilità clinica e quella gestionale devono coincidere. Il singolo paziente deve essere assegnato all'Unità Operativa del medico responsabile.

In considerazione dell'ampia variabilità di problematiche cliniche e dell'elevata complessità e polipatologicità della maggior parte dei pazienti, con connotazioni di tipo prevalentemente internistico o di specialità affini, è auspicabile che la responsabilità clinico-gestionale sia affidata preferibilmente a un dirigente medico in possesso di conoscenze ed esperienze di tipo internistico, con il compito di effettuare la doverosa sintesi dei problemi e di collocare e coordinare in maniera idonea la presenza e la consulenza dei diversi specialisti afferenti.

L'organizzazione del lavoro, in équipe, multidisciplinare e multiprofessionale, deve essere adeguatamente regolamentata e strutturata con la previsione di orari per i momenti di visita, per i momenti di briefing e di confronto, per l'attivazione e la presenza di specialisti diversi dalle équipe curanti.

La visita medica e i momenti di briefing e condivisione devono prevedere la presenza di più operatori, medici e infermieri in numero adeguato alle esigenze.

Per il personale infermieristico devono essere previsti compiti ben definiti e tali da contemplare possibili aree e ambiti di autonomia prevalentemente di tipo clinicoassistenziale e educazionale.
Per favorire un corretto funzionamento e una gestione integrata ed efficace delle diverse Unità Operative afferenti, può essere opportuno che nelle aree comprendenti un maggior numero di letti (area acuti e area post-acuti) vengano identificati settori ove allocare e accorpare, pur nell'ottica di una massima flessibilità, i pazienti assegnati a ogni singola Unità Operativa.

Questo soprattutto per favorire un ordine e una consequenzialità nell'organizzazione quotidiana dell'attività e in particolare nel momento, ritenuto da noi ancora essenziale nella gestione dei pazienti ospedalizzati, della visita medica.

Il Consiglio Direttivo FADOI Emilia Romagna Giorgio Cioni (Presidente - Pavullo, MO) Giovanni losa (Vicepresidente - Cesenatico, FC) Ido Iori (Past President FADOI Nazionale - Reggio Emilia)

Giuseppe Civardi (Past President - Fiorenzuola, PC)

Giuseppe Chesi* (Past President - Scandiano, RE) Maurizio Ongari (Segretario - Porretta Terme, BO) Giorgio Ballardini (Tesoriere - Rimini)

Domenico Panuccio (Segretario FADOI Nazionale - Bologna)

Roberto Nardi (Editor in Chief Italian Journal of Medicine - Bologna) Esterita Accogli (Consigliere - Bologna) Gianpaolo Bragagni (Consigliere San Giovanni in Persiceto, BO) Fabio Gilioli (Consigliere - Carpi, MO) Matteo Giorgi Pierfranceschi (Consigliere - Piacenza) Pasquale Gianluca Giuri (Referente FADOI Area Giovani Emilia Romagna - Castelnuovo ne' Monti, BO) Marco Grandi (Consigliere - Sassuolo, MO) Loris Montanari (Consigliere - Ravenna) Maurizio Nizzoli (Consigliere - Forli)

Pedretti Giovanni (Consigliere - Fidenza, PR) Mauro Silingardi (Consigliere - Guastalla, RE)

Daniela Tirotta (Consigliere - Cattolica, RN) Tiziana Montruccoli (Presidente ANIMO - Scandiano, RE)

${ }^{*}$ Email: chesig@ausl.re.it (G. Chesi).

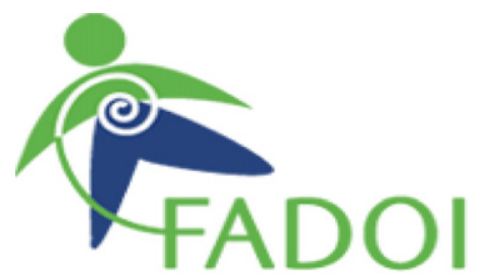

FEDERAZIONE DELLE ASSOCIAZIONI DEI DIRIGENTI OSPEDALIERI INTERNISTI 